\title{
HIF-1a Inhibits Caspase 3 Mediated Apoptosis and Promotes Tumor Growth of Prostatic Carcinoma through Up Regulation of Cyclophilin A.
}

Mariana F Gayyed ${ }^{1 *}$, Medhat M. Soliman ${ }^{2}$, Ahmed Zaki Mohamed Anwer ${ }^{3}$, Ehab Rifat Tawfiek ${ }^{4}$, Nehad M. Reda AbdelMaqsoud $^{5}$

\author{
${ }^{1}$ Assistant professor of Pathology, Faculty of Medicine, Minia University, Minia, Egypt \\ ${ }^{2}$ Consultant of Onco-surgery, Minia Oncology Center, Minia, Egypt \\ ${ }^{3}$ Professor of Urology, Faculty of Medicine, Minia University, Minia, Egypt \\ ${ }^{4}$ Professor of Urology, Faculty of Medicine, Minia University, Minia, Egypt \\ ${ }^{5}$ Professor of Pathology, Faculty of Medicine, Minia University, Minia, Egypt
}

\begin{tabular}{ll}
\hline DOI: $\underline{10.36348 / \text { sjpm.2020.v05i12.002 }}$ & | Received: 15.11 .2020 | Accepted: $23.11 .2020 \mid$ Published: 10.12 .2020 \\
*Corresponding author: Mariana F Gayyed &
\end{tabular}

\section{Abstract}

Background: The management of prostate cancer may be difficult due to apoptosis resistance. HIF-1 $\alpha$ (Hypoxia inducible factor $1 \alpha$ ) and CypA (cyclophilin A) over expression promotes this resistance. Caspase 3 is a central executioner of the apoptosis pathway. Changed expression may correspond to tumor cell challenge. Aim: Evaluation of HIF-1 $\alpha$, CypA and Caspase 3 expressions in BPH (benign prostatic hyperplasia), PIN (prostatic intra-epithelial neoplasia) and PCa (prostatic carcinoma). Methods: One hundred four patients with histologically diagnosed prostatic carcinoma, 20 cases with BPH and 24 cases with PIN were investigated. Immunohistochemical assessment was executed to evaluate HIF-1 $\alpha$, CypA and Caspase 3 expressions. Results: In BPH cases, HIF-1 $\alpha$, CypA and Caspase 3 were expressed in 20\%, 30\% and 60\% respectively. In PIN cases, HIF-1 $\alpha$, CypA and Caspase 3 were expressed in $33.3 \%$, $41.7 \%$ and $41.7 \%$, respectively while in PCa cases, HIF-1 $\alpha$, CypA and Caspase 3 were expressed in 57.7\%, 69.2\% and $25 \%$ respectively. Increasing stage of prostatic cancer showed a significant increased HIF- $1 \alpha$ and CypA immunoexpression with lost caspase 3 immuno-expressions. Conclusions: Distorted HIF-1 $\alpha$, CypA and Caspase 3 expressions may act as a further mechanism of apoptotic resistance in prostatic carcinoma treatment.

Keywords: HIF-1 $\alpha$; CypA; Caspase 3; prostatic carcinoma.

Copyright (C) 2020 The Author(s): This is an open-access article distributed under the terms of the Creative Commons Attribution 4.0 International License (CC BY-NC 4.0) which permits unrestricted use, distribution, and reproduction in any medium for non-commercial use provided the original author and source are credited.

\section{INTRODUCTION}

Prostate carcinoma ( $\mathrm{PCa}$ ) is a commonly diagnosed malignant tumor in males, worldwide. Among Egyptian men, it is considered the fifth most common type of cancer [1]. Hypoxia is a corner stone in initiation, and evolution of prostate cancer. The main task of hypoxia is the alteration of genes, leading to malignant progression [2]. HIFs (the hypoxia-inducible factors) mediate the effect of hypoxia on tumor cells [3, 4]. HIF-1 $\alpha$ controls multiple hypoxia-inducible genes included in tumor proliferation, and metastasis. Poor prognosis in most cancers was associated with increased HIF-1 $\alpha[5,6]$.

CypA (Cyclophilin A) is one of the immunophilin family members that protects the cells via binding to cyclosporine A [7, 8]. Even with the intracellular localization of CypA, some cells could secrete it in reaction to inflammation and tumor hypoxia $[9,10]$. CypA is essential in protein formation and impacts the maintenance of partially destructed proteins during environmental misuses [11]. CypA overexpression is detected in breast carcinoma $[12,13]$, lung carcinoma $[14,15]$, gastric carcinoma [16], pancreatic cancer [17], cholangiocarcinoma [18], carcinoma of the liver [19], carcinoma of the kidney [20], carcinoma of the colon [21], and glioblastoma [22]. Although the title role of CypA in programmed cell death is uncertain, several studies show that CypA is liberated in reaction to hypoxia and may act as cytoprotective against oxidative stress-induced apoptosis [23].

The family of caspase is a protease that plays a central role in the apoptosis. Active Caspase 3 is an essential marker for apoptosis. During carcinogenesis, Caspase 3 expression may cause cell resistance during apoptosis. For this reason, Caspase immuno-expression is behaved as a marker for solid tumors development, as well as aseek out curative management [24]. 
Still, the association of HIF-1 $\alpha$, CypA and Caspase 3 expressions with clinicopathological features is unsettled. Our aim is to explore HIF- $1 \alpha-$ CypACaspase 3 pathway in benign prostatic hyperplasia $(\mathrm{BPH})$, premalignant prostatic lesion such as prostatic intraepithelial neoplasia (PIN) and PCa in addition to demonstrate the association of HIF-1 $\alpha$, CypA and Caspase 3 immunohistochemical expressions with clinicopathological features in patients with PCa.

\section{MATERIALS AND METHODS}

We have studied 104 cases of prostatic carcinomas, collected from the archives of the Pathology Departments of both Minia Oncology Center and Minia University Hospital, Egypt. Additionally, 20 cases of BPH and 24 cases of PIN were involved in the study. All the samples were collected between 2010 to 2018. All specimens had a confirmed pathological diagnosis and were classified following the WHO classification (4th Edition, 2015). Patients did not take chemotherapy, radiotherapy, or targeted therapy. Ethical approval was taken from the ethical committees of both Faculty of Medicine, Minia University, and Minia Oncology Center.

\section{Immunohistochemistry}

Sections $4 \mu \mathrm{m}$ thick were subjected to deparaffinization in xylene, rehydration through a graded ethyl alcohol. Immunohistochemistry staining was executed via the HRP/DAB detection immunohistochemistry kit (ab64264, Abcam)). Endogenous peroxidase activity was blocked by using $3 \%$ hydrogen peroxide for 10 minutes. After rinsing in phosphate-buffered saline (PBS), citrate buffer ( $\mathrm{pH}$ 6.0) was used as an antigen retrieval (microwave heating for 15 minutes). After cooling at room temperature, sections were incubated with primary antibodies for HIF-1 $\alpha$ (Invitogen, 1:100), CypA (Invitrogen, 1:100) and Caspase 3 (Abcam, 1:100) at $4{ }^{\circ} \mathrm{C}$ overnight. After rinsing in $\mathrm{PBS}$, the biotinylated antipolyvalent was added at $37{ }^{\circ} \mathrm{C}$ for 30 minutes followed by incubation with streptavidin peroxidase for thirty minutes. Incubation with DAB (diaminobenzidine) and counterstaining with Mayer's haematoxylin was performed. Finally, slides were dehydrated, and cover slipped.

\section{Evaluation of immunohistochemistry}

The scoring of immunohistochemical (IHC) staining was accomplished by the two pathologists. The IHC score of HIF-1 $\alpha$, CypA and Caspase 3 was built on intensity, and percentage of positively stained cells. The intensity staining score was assigned as 0 (no staining), 1 (weak staining), 2 (moderate staining), and 3 (strong staining). The proportion staining score was assigned as 0 ( $<5 \%$ positive cells), 1 (6-25\% positive cells), 2 (26$50 \%$ positive cells), 3 (51-75\% positive cells), and 4 (>75\% positive cells). The final staining score was assumed by multiplication of the intensity and percentage scores: 0 (negative), 1 (1-4), 2 (5-8), and 3
(9-12). A final immunohistochemical staining score of 0 or 1 was combined into the low immunohistochemical expression, and a final score of 2 or 3 was combined into the high immunohistochemical expression [24].

\section{STATISTICAL ANALYSIS}

Statistical analysis was performed using SPSS 22.0 software. Chi-square and Fischer's exact tests were used to investigate the significance of relationship between markers, and the individual variables. Person correlations test was used to investigate the correlations between immunohistochemical markers in different lesions. The overall survival (OS) curves were assessed using the Kaplan Meier method. A multivariate analysis was carried out using Cox's regression model. P values $\leq 0.05$ were of statistical significance.

\section{RESULTS \\ Clinicopathological data of patients}

The mean age of BPH was $65.50 \pm 7.90$ years and a median of 65 years (range 50-78 years). The mean age of the examined PIN cases was $69.08 \pm 4.35$ years and a median of 69.50 years (range 61-75 years). Eight cases (33.3\%) showed LGPIN and 16 cases $(66.7 \%)$ HGPIN

The mean age of prostatic carcinoma was $67.67 \pm 6.74$ years and a median of 68 years (range 49 82 years). Prostate-specific antigen (PSA) was 22 cases $(21.2 \%)$ less than 10,50 cases $(48.1 \%)$ between 10 - 20 and 32 cases $(30.8 \%)$ more than 20 . The Gleason scores (GS) were as follow: 28 cases $(26.9 \%)$ were Gleason score $2-6,46$ cases $(44.2 \%)$ were Gleason score 7 , and 30 cases $(28.8 \%)$ was Gleason score $8-10$. Pathological tumor stage (pT) was pT2 in 58 cases $(55.8 \%)$, pT3 in 34 cases (32.7\%) and pT4 in 12 cases (11.5\%). Perineural invasion was present in 56 cases $(55.8 \%)$ and absent in 48 cases $(46.2 \%)$. Lymph node metastasis (LNM) was absent in 80 cases (76.9\%) and found in 24 cases $(23.1 \%)$. Regarding the stage, 8 cases $(7.7 \%)$ were stage I, $22(21.2 \%), 18(17.3 \%)$ cases were stages IIA, IIB respectively, stage III were $24(23.1 \%)$ cases, and stage IV 32 were $(30.8 \%)$ cases. Table 1.

\section{Immunohistochemical expression in the studied cases}

\section{HIF-1 $\alpha$ immunohistochemical expression}

In BPH, HIF-1 $\alpha$ was high expression in 4 cases $(20 \%)$ and low expression in 16 cases $(80 \%)$.

In PIN, HIF-1 $\alpha$ was high expression in 8 cases $(33.3 \%)$ and low expression in 16 cases $(66.7 \%)$. In LGPIN and HGPIN, HIF-1 $\alpha$ was high expression in 2 case $(25 \%)$ and 6 cases $(37.5 \%)$ respectively and the difference does not reach a significant level $(\mathrm{P}=594)$.

In $\mathrm{PCa}$ cases $\mathrm{HIF}-1 \alpha$ was high expression in 60 cases $(57.7 \%)$ and low expression in 44 cases $(42.3 \%)$. Figure 1. Significant statistically changess were present among the three groups $(\mathrm{P}=0.047)$, however there was no statistically significant 
differences between $\mathrm{BPH}$ vs $\mathrm{PIN}$ or $\mathrm{PCa}(\mathrm{P}=0.667$ ,0.622 respectively) and $\mathrm{PIN}$ vs $\mathrm{PCa}(\mathrm{P}=0.745)$.

\section{CypA immunohistochemical expression}

BPH showed negative/weak immunoexpression in $14(70 \%)$ cases while $6(30 \%)$ of cases showed positive expression. PIN cases were negative/ weak expression in $14(58.3 \%)$ cases and $10(41.7 \%)$ of cases showed positive expression. LGPIN showed negative/ weak in $4(50 \%)$ cases and $4(50 \%)$ cases show positive expression while HGPIN showed 10 $(62.5 \%)$ negative/ weak and 6 (37.5\%) positive expression. There were no statistically significant differences in CypA expression between LGPIN and HGPIN ( $\mathrm{P}=0.576)$.

As regarded carcinoma cases we found negative/ weak expression in $32(30.8 \%)$ cases and 72 $(69.2 \%)$ of cases showed positive expression, figure 1 .

Statistically significant differences were present among the three groups $\mathrm{BPH}$ vs PIN vs $\mathrm{PCa}$ $(\mathrm{P}=0.027)$ and between PIN vs $\mathrm{PCa}(\mathrm{P}=0.010)$ while there was no statistically significant differences between $\mathrm{BPH}$ vs PIN ( $\mathrm{P}=0.183)$ and $\mathrm{BPH}$ vs $\mathrm{PCa}$ $(\mathrm{P}=0.183)$.

\section{Caspase 3 immunohistochemical expression}

In BPH cases, Caspase 3 expression was positive in 12 cases $(60 \%)$ and negative in 8 cases $(40 \%)$.

In PIN cases, Caspase 3 positive expression in 10 cases $(41.7 \%)$ and negative expression 14 cases (58.3\%). In LGPIN and HGPIN, Caspase 3 was positive expression in 5 case $(62.5 \%)$ and 5 cases $(31.3 \%)$ respectively and the difference does not reach a significant level $(\mathrm{P}=153)$. In $\mathrm{PCa}$ cases, Caspase 3 was positive expression in 26 cases $(25 \%)$ and negative in 78 cases $(75 \%)$, figure 1 .

A statistically significant changes were present among the three groups $(\mathrm{P}=0.05)$, however there was no statistically significant differences between BPH or PIN vs carcinoma $(\mathrm{P}=0.535, \mathrm{p}=0.668$ respectively $)$ and $\mathrm{BPH}$ vs PIN ( $\mathrm{P}=0.612)$.

\section{The HIF-1a, CypA and Caspase 3 immunohistochemical expression and clinicopathological issues}

Table 2 summarizes the immunohistochemical expressions of HIF-1 $\alpha$, CypA and Caspase 3 expressions in relation to different clinicopathological issues in PCa cases.

There were statistically significant associations between HIF-1 $\alpha$ expression and perineural invasion, LNM (pN status) $(\mathrm{P}=0.029, \mathrm{p}=0.008$ respectively). No significant associations were seen with other clinicopathological issues.
A statistically significant associations between CypA expression and Gleason score, pathological $\mathrm{T}$ (pT) stage, LNM (pN status) and stage were identified ( $\mathrm{P}=0.031, \mathrm{p}=0.008, \mathrm{p}=0.006$ and $\mathrm{p}=0.011$ respectively). No significant associations were seen in relation to patients' age, PAS and perineural invasion.

A statistically significant associations between Caspase 3 expression and Gleason score, pathological T (pT) stage, LNM (pN status) and stage were identified ( $\mathrm{P}=0.013, \mathrm{p}=0.003, \mathrm{p}=0.024$ and $\mathrm{p}<0.001$ respectively). No significant associations were seen in relation to patients' age, PAS and perineural invasion.

A statistically significant relationship between HIF- $1 \alpha$ expression and OS was identified $(\mathrm{P}<0.001)$. A statistically significant relationship between CypA expression and OS was identified $(\mathrm{P}<0.001)$. A statistically significant relationship between Caspase 3 expression and OS was identified $(\mathrm{P}<0.001)$.

\section{Associations between examined markers in different examined lesions}

Associations between immunohistochemical expressions of the markers in different examined lesions was shown in table 3.

In $\mathrm{BPH}$ cases, a statistically significant correlation was noted between CypA/ HIF-1 $\alpha$ expression $(\mathrm{r}=0.489, \mathrm{P}=0.025)$. There was an inverse correlation between CypA/ Caspase 3 but not statistically significant $(\mathrm{r}=0.293, \mathrm{P}=0.211)$. Also, there was an inverse correlation between HIF-1 $\alpha /$ Caspase 3 but not statistically significant $(r=-0.093, \mathrm{P}=0.696)$.

In PIN cases, No statistically significant correlation was noted between examined markers, HIF$1 \alpha /$ CypA expression $(\mathrm{r}=0.238, \mathrm{P}=0.263)$, between CypA/ Caspase $3(r=-0.290, P=0.169)$ and HIF-1 $\alpha$ / Caspase $3(\mathrm{r}=-0.265, \mathrm{P}=0.211)$.

In PCa cases, a statistically significant positive correlation was noted between HIF-1 $\alpha /$ CypA expression $(\mathrm{r}=0.792, \mathrm{P}<0.001)$. An inverse statistically significant correlation was noted between CypA/ Caspase $3(\mathrm{r}=-0.354, \mathrm{P}<0.001)$ and HIF-1 $\alpha /$ Caspase 3 $(\mathrm{r}=-0.361, \mathrm{P}<0.001)$.

\section{Prognostic significance and survival assessment}

The time of overall survival ranged from 4 months to 60 months with a mean ( \pm standard deviation; SD) of $28.08( \pm 15.27)$ months and a median survival time was of 24 months. The overall survival was $38.5 \%$.

High Gleason score $(\mathrm{P}=0.001), \mathrm{pN}$ status $(\mathrm{P}=0.042)$, perineural invasion $(\mathrm{P}=0.047)$, and high stage $(\mathrm{P}=0.040)$ were the only adverse prognostic clinicopathological factors. While overall survival rate 
did not significantly influence by patients' age $(\mathrm{P}=0.397)$, PSA $(\mathrm{P}=0.249)$ and $\mathrm{pT}$ status $(\mathrm{P}=0.090)$.

Regarding overall survival and examined markers; increased expression HIF- $1 \alpha$ and CypA were associated with shorter OS $(\mathrm{P}=0.024,<0.001$ respectively), while low Caspase 3 immuno-expression was associated with shorter OS $(\mathrm{P}=0.023)$

Regarding the multivariate analysis, stage, HIF- $1 \alpha$ and CypA continued to be a significant prophet of OS $(\mathrm{P}<0.001$ for each Table 4

Table-1: Patients' clinicopathological parameters of cases $(\mathrm{N}=104)$

\begin{tabular}{|l|l|l|l|}
\hline Clinicopathological parameters & No & $\mathbf{\%}$ \\
\hline \multirow{4}{*}{ Age } & $\leq \mathbf{6 0}$ & 10 & 9.6 \\
\cline { 2 - 4 } & $\mathbf{7 6 0}$ & 94 & 90.4 \\
\hline \multirow{4}{*}{ PSA } & $<\mathbf{1 0}$ & 22 & 21.2 \\
\cline { 2 - 4 } & $\mathbf{1 0 - 2 0}$ & 50 & 48.1 \\
\cline { 2 - 4 } & $\mathbf{2 0}$ & 32 & 30.8 \\
\hline \multirow{4}{*}{ Gleason score } & Gleason 2-6 & 28 & 26.9 \\
\cline { 2 - 4 } & Gleason 7 & 46 & 44.2 \\
\cline { 2 - 4 } & Gleason 8-10 & 30 & 28.8 \\
\hline \multirow{4}{*}{ pT status } & T2 & 58 & 55.8 \\
\cline { 2 - 4 } & T3 & 34 & 32.7 \\
\cline { 2 - 4 } & T4 & 12 & 11.5 \\
\hline \multirow{3}{*}{ pN status } & Negative & 48 & 46.2 \\
\cline { 2 - 4 } & Positive & 56 & 53.8 \\
\hline \multirow{4}{*}{ Stage } & No & 80 & 76.9 \\
\cline { 2 - 4 } & N1 & 24 & 23.1 \\
\hline & I & 8 & 7.7 \\
\cline { 2 - 4 } & IIA & 22 & 21.2 \\
\cline { 2 - 4 } & IIB & 18 & 17.3 \\
\cline { 2 - 4 } & III & 24 & 23.1 \\
\cline { 2 - 4 } & IV & 32 & 30.8 \\
\hline \multirow{3}{*}{ DFS } & Free & 40 & 38.5 \\
\cline { 2 - 4 } & Recurrence & 64 & 61.5 \\
\hline
\end{tabular}

pT status: Pathological tumor stage

pN: Lymph node status

DFS: Disease free Survival

Table-2: Association of CypA, HIF-1 $\alpha$ and Caspase 3 immunohistochemical expressions with clinicopathological characters in prostatic carcinoma $(n o=104)$

\begin{tabular}{|c|c|c|c|c|c|c|c|c|c|c|}
\hline \multirow{2}{*}{\multicolumn{2}{|c|}{ Clinicopathological parameters }} & \multicolumn{3}{|c|}{ HIF-1 $\alpha$ expression } & \multicolumn{3}{|c|}{ CypA expression } & \multicolumn{3}{|c|}{ Caspase 3 expression } \\
\hline & & Low (\%) & High (\%) & P-value & Low $(\%)$ & High $(\%)$ & P-value & Low (\%) & High $(\%)$ & P-value \\
\hline \multirow{2}{*}{ Age } & $\leq \mathbf{6 0}$ & $4(40)$ & $6(60)$ & \multirow{2}{*}{0.648} & $2(20)$ & $8(80)$ & \multirow{2}{*}{0.508} & $8(80)$ & $2(20)$ & \multirow{2}{*}{0.522} \\
\hline & $>60$ & $4042.6)$ & $54(57.4)$ & & $30(31.9)$ & $64(68.1)$ & & $70(74.5)$ & $24(25.5)$ & \\
\hline \multirow{3}{*}{ PSA } & $<10$ & $12(54.5)$ & $10(45.5)$ & \multirow{3}{*}{0.578} & $10(45.5)$ & $12(54.5)$ & \multirow{3}{*}{0.438} & $15(68.2)$ & $7(31.8)$ & \multirow{3}{*}{0.691} \\
\hline & $10-20$ & $18(36)$ & $32(64)$ & & $12(24)$ & $38(76)$ & & $38(76)$ & $12(24)$ & \\
\hline & $>20$ & $14(43.7)$ & $18(56.3)$ & & $10(31.3)$ & $22(68.8)$ & & $25(78.1)$ & $7(21.9)$ & \\
\hline \multirow{3}{*}{ Gleason score } & Gleason 2-6 & $18(64.3)$ & $10(35.7)$ & \multirow{3}{*}{0.054} & $16(57.1)$ & $12(42.9)$ & \multirow{3}{*}{0.031} & $17(60.7)$ & $11(39.3)$ & \multirow{3}{*}{0.013} \\
\hline & Gleason 7 & $20(43.5)$ & $26(56.5)$ & & $12(26.1)$ & $34(73.9)$ & & $33(71.7)$ & $13(28.3)$ & \\
\hline & Gleason 8-10 & $6(20)$ & $24(80)$ & & $4(13.3)$ & $26(86.7)$ & & $28(93.3)$ & $2(607)$ & \\
\hline \multirow{3}{*}{ pT status } & T2 & $28(48.3)$ & $30(51.7)$ & \multirow{3}{*}{0.359} & $28(48.3)$ & $30(51.7)$ & \multirow{3}{*}{0.008} & $36(62.1)$ & $22(37.9)$ & \multirow{3}{*}{0.003} \\
\hline & T3 & $14(41.2)$ & $20(58.8)$ & & $4(11.8)$ & $30(88.2)$ & & $31(91.2)$ & $3(8.8)$ & \\
\hline & T4 & $2(16.7)$ & $10(83.3)$ & & $4(44.4)$ & $8(55.6)$ & & $11(91.7)$ & $1(8.3)$ & \\
\hline \multirow{2}{*}{ Perineural Invasion } & Negative & $28(58.3)$ & $20(41.7)$ & \multirow{2}{*}{0.029} & $18(37.5)$ & $30(62.5)$ & \multirow{2}{*}{0.251} & $34(70.8)$ & $14(29.2)$ & \multirow{2}{*}{0.248} \\
\hline & Positive & $16(28.6)$ & $40(71.4)$ & & $14(25)$ & $42(75)$ & & $44(78.6)$ & $12(21.4)$ & \\
\hline \multirow{2}{*}{ pN status } & No & $42(52.5)$ & $38(47.5)$ & \multirow{2}{*}{0.006} & $32(40)$ & $4860)$ & \multirow{2}{*}{0.006} & $56(70)$ & $24(30)$ & \multirow{2}{*}{0.024} \\
\hline & N1 & $2(8.3)$ & $22(91.7)$ & & $0(0)$ & $24(100)$ & & $22(91.7)$ & $2(8.3)$ & \\
\hline \multirow{5}{*}{ Stage } & I & $6(75)$ & $2(25)$ & \multirow{5}{*}{0.173} & $6(75)$ & $2(25)$ & \multirow{5}{*}{0.011} & $1(12.5)$ & $7(78.5)$ & \multirow{5}{*}{$<0.001$} \\
\hline & IIA & $12(54.5)$ & $10(45.5)$ & & $12(54.5)$ & $10(45.5)$ & & $15(68.2)$ & $7(31.8)$ & \\
\hline & IIB & $8(44.4)$ & $10(55.6)$ & & $8(44.4)$ & $10(55.6)$ & & $13(72.2)$ & $5(27.8)$ & \\
\hline & III & $12(50)$ & $12(50)$ & & $4(16.7)$ & $20(83.3)$ & & $22(91.7)$ & $2(8.3)$ & \\
\hline & IV & $6(18.8)$ & $26(81.3)$ & & $2(6.3)$ & $30(93.8)$ & & $27(84.4)$ & $5(15.6)$ & \\
\hline OS & Live & $28(70)$ & $12(30)$ & $<000$ & $30(75)$ & $10(25)$ & $<0$ & $20(50)$ & $20(50)$ & \\
\hline & Death & $16(25)$ & $48(75)$ & $<0.00$ & $2(3.1)$ & $62(96.9)$ & $<0.0$ & $58(90.6)$ & $6(9.4)$ & $<0$ \\
\hline
\end{tabular}

Test of significance: Chi-squared and Fischer's exact tests

$p$-value $<0.05$ is considered significant.

pT status: Pathological tumor stage

pN: Lymph node status

OS: Overall Survival 
Table-3: Correlations between CypA, HIF-1 $\alpha$ and Caspase 3 in the studied prostatic lesions:

\begin{tabular}{|c|c|c|c|c|c|}
\hline Lesion & Marker expression & & 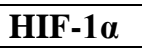 & СурА & Caspase 3 \\
\hline \multirow{6}{*}{$\underset{n}{a t}$} & \multirow[t]{2}{*}{ HIF-1a } & Pearson Correlation & - & $0.498 *$ & -0.093 \\
\hline & & $\mathrm{P}$ value & - & 0.025 & 0.696 \\
\hline & \multirow[t]{2}{*}{ СурА } & Pearson Correlation & $0.498^{*}$ & - & -0.293 \\
\hline & & $\mathrm{P}$ value & 0.025 & - & 0.211 \\
\hline & \multirow[t]{2}{*}{ Caspase 3} & Pearson Correlation & -0.093 & -0.293 & - \\
\hline & & $\mathrm{P}$ value & 0.696 & 0.211 & - \\
\hline \multirow{6}{*}{ Z } & \multirow[t]{2}{*}{ HIF-1 $\alpha$} & Pearson Correlation & - & 0.238 & $0.265-$ \\
\hline & & $\mathrm{P}$ value & - & 0.263 & 0.211 \\
\hline & \multirow[t]{2}{*}{ СурА } & Pearson Correlation & 0.238 & - & $0.290-$ \\
\hline & & $\mathrm{P}$ value & 0.263 & - & 0.169 \\
\hline & \multirow[t]{2}{*}{ Caspase 3} & Pearson Correlation & $0.265-$ & $0.290-$ & -- \\
\hline & & $\mathrm{P}$ value & 0.211 & 0.169 & - \\
\hline \multirow{6}{*}{ 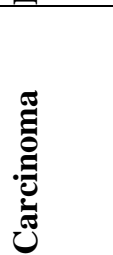 } & \multirow[t]{2}{*}{ HIF-1 $\alpha$} & Pearson Correlation & - & $0.792 * *$ & $0.361 * *_{-}$ \\
\hline & & $\mathrm{P}$ value & - & $<0.001$ & $<0.001$ \\
\hline & \multirow[t]{2}{*}{ СурА } & Pearson Correlation & $0.792 * *$ & - & $0.354 * *_{-}$ \\
\hline & & $\mathrm{P}$ value & $<0.001$ & - & $<0.001$ \\
\hline & \multirow[t]{2}{*}{ Caspase 3} & Pearson Correlation & $0.361 * *_{-}$ & $0.354 * *_{-}$ & - \\
\hline & & $\mathrm{P}$ value & $<0.001$ & $<0.001$ & - \\
\hline
\end{tabular}

**Test of significance: Pearson correlation

*Correlation is significant at the 0.05 level ( $\mathrm{p}$ value).

Table-4: Multivariate analyses of overall survival in prostate carcinoma patients

\begin{tabular}{|l|l|l|l|}
\hline Variables & HR & $\mathbf{9 5 \%}$ CI & P value \\
\hline Grade & 0.836 & $0.555-1.258$ & 0.398 \\
\hline pN status & 0.553 & $0.285-1.071$ & 0.079 \\
\hline Perineural invasion & 1.268 & $0.811-1.982$ & 0.298 \\
\hline Stage & 1.677 & $1.335-2.108$ & $<\mathbf{0 . 0 0 1}$ \\
\hline HIF-1a & 3.404 & $1.819-6.374$ & $<\mathbf{0 . 0 0 1}$ \\
\hline CypA & 1.386 & $1.196-2.759$ & $<\mathbf{0 . 0 0 1}$ \\
\hline Caspase 3 & 0.729 & $0.426-1.248$ & 0.249 \\
\hline
\end{tabular}




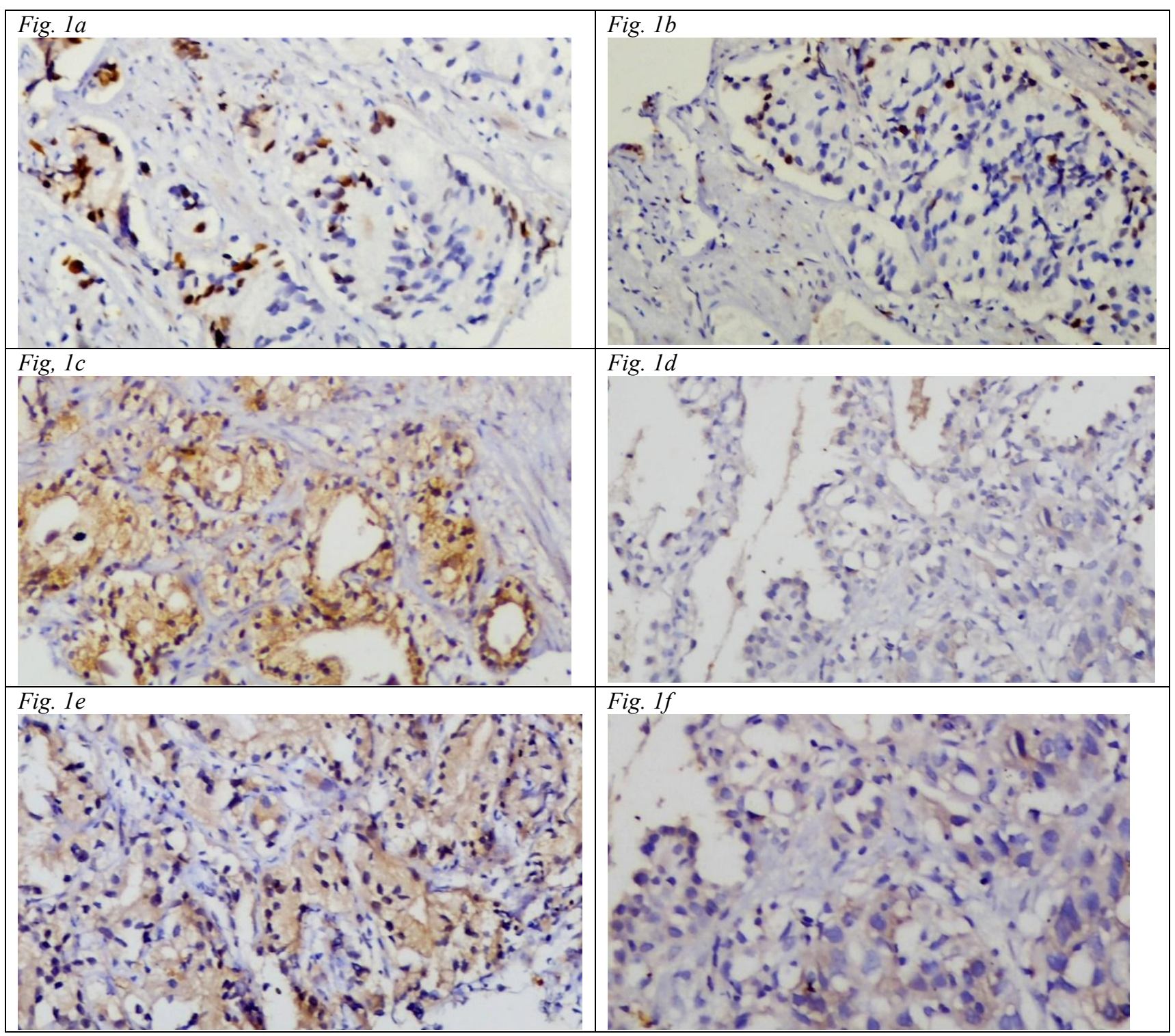

Fig-1: HIF-1a, CypA and Caspase 3 immunohistochemical expressions in prostatic carcinoma. (a) high HIF-1 $\alpha$ immune-expression in PCa, (b) low HIF-1 $\alpha$ immune-expression in PCa, (c) high CypA immune-expression in PCa, (d) low CypA immune-expression in PCa, (e) high Caspase 3 immune-expression in PCa, (f) low Caspase 3 immune-expression in PCa. Magnification 40x.

\section{DISCUSSION}

Stress resistance is an essential character of cancer cells of solid tumors. These stresses involve hypoxia, chemotherapeutic agents, and radiotherapy. Many defense mechanisms are used by cancer cells against these stresses. One of these mechanisms is induction of HIF-1 $\alpha$ under hypoxic conditions. It makes cancer cells to survive by regulation of hypoxia-induced apoptotic cell death. Thus, it promotes metastasis by controlling of some factors including CypA [25].

The family of cyclophilin is comprised of cyclophilins A, B, C, and D. The classic member of this family preserved in mammalian cells is CypA [26]. CypA, is the cytosolic receptor for an immunosuppressant drug, the cyclosporin A [26, 27]. CypA is overexpressed in many cancers, including breast [12], pancreatic [17], colorectal [21], and lung $[14,15]$ cancers.
The control of CypA expression occurred through the direct junction between HIF-1 $\alpha$ and CypA promoter regions. Increased expression of HIF- $1 \alpha$ and CypA supported cell proliferation, invasion, and suppressed apoptosis [25].

Overexpression of HIF-1 $\alpha$ in many solid tissue cancers is evidenced by many researches, including carcinoma of ovary, esophagus, breast, urinary bladder, and oral cavity [28, 29, 30, 31]. Various studies showed that HIF-1 $\alpha$ [32] and caspase-3 [33] are upregulated in prostatic carcinoma compared with non-tumor tissue. On the other hand, no study deals with the simultaneous expression of HIF-1 $\alpha$, CypA and Caspase 3, or assessed the relations between their expression and the clinicopathological features in PCa. 
For the first time, we examined the protein expressions of HIF-1 $\alpha$, CypA and Caspase 3 in 104 PCa, 24 PIN and 20 BPH using immunohistochemistry to illustrate the correlation between HIF-1 $\alpha$, CypA and Caspase 3.

We noticed that the expression of HIF-1 $\alpha$ in $\mathrm{PCa}$ cases was significantly higher than those of BPH and PIN cases. The differences among the three groups were statistically significant, compared to the differences between BPH vs PIN or PCa and PIN vs PCa. Also, HIF-1 $\alpha$ expression was significantly correlated with perineural invasion, LNM ( $\mathrm{pN}$ status). No significant relations were detected with other clinicopathological characters. This finding was coincided with that reported with $\mathrm{Ni}$ et al. [34], who found that high HIF-1 $\alpha$ protein expression was significantly related to LNM. However, Weranja et al. [25], stated that high HIF-1 $\alpha$ expression was significantly high with tumor stage in PCa cases.

We found that CypA is detectable in most PCa cases and only a small set of BPH and PIN. Significantly higher CypA expression is present in $\mathrm{PCa}$ tissues than in BPH and PIN, indicating that CypA is up regulated in the $\mathrm{PCa}$ transformation. Significant differences were found among the BPH vs PIN vs PCa groups and between PIN vs PCa groups. There were no significant associations between BPH vs PIN and BPH vs PCa. This finding was like that is stated by Min et al. [35], who reported high expression of CypA in pancreatic carcinoma in comparison to normal pancreatic tissue. In addition, CypA expression significantly correlated with the Gleason score, pathological Tumor (pT) stage, LNM (pN status) and TNM stage, as well as the level of HIF-1 $\alpha$. No significant correlations were seen regarding patients' age, PAS level and perineural invasion. These findings recommended that CypA may be involved in the growth, and progression of PCa. Previous immunohistochemical assay indicated that CypA was highly expressed in esophageal carcinoma including tumor grade correlation [36]. Researchers found that CypA levels were significantly higher in NSCLC cell lines than in normal lung cell lines [37]. The elevation of CypA protein level in PCa tissue specimens in the present study has coincided with other studies, exploring the primary role of CypA in tumorigenesis [19].

CypA overexpression is related to increase HIF-1 $\alpha$ expression. The association between increased expression of CypA and HIF-1 $\alpha$ was settled by the finding of Kyo et al. [38], who stated that HIF-1 $\alpha$ transcription factor initiated CypA promoter and this in turn caused up-regulation of CypA. In pancreatic carcinoma, researchers showed that the downstream gene of HIF-1 $\alpha$ is CypA and the high expression of both genes was significantly associated with LNM and high tumor stage [21].
Regarding Caspase 3 expression, we found that the expression of caspase 3 was more in $\mathrm{BPH}$ than PIN and PCa. The three studied groups expressed statistically significant differences nevertheless the differences between BPH or PIN vs carcinoma and BPH vs PIN were not statistically significant. Sohn et al. found that Caspase 3 immuno-expression in the cases of BPH were higher than its expression in grade III PCa cases [39]. Also, O' Neill et al. reported that the expression of Caspase 3 was decreased in high-grade PCa compared to BPH [24]. In Prostatic carcinoma cases, we also observed a statistically significant associations between Caspase 3 expression, and Gleason score, pathological tumor (pT) stage, LNM (pN status), and TNM stage. This was correlated with what was found by O' Neill et al. [24]. Similarly, in the study of Winter et al., Caspase 3 expression was decreased in prostatic carcinoma, high grade than in normal prostate [40]. No significant relations regarding patients' age, PAS and perineural invasion. In this study, Caspase 3 expression was inversely associated with HIF-1 $\alpha$ and CypA expressions.

Our study is the first to detect that the expression level of activated Caspase 3 staining in HGPIN lesions was lower than that in LGPIN and BPH.

In our study, high HIF-1 $\alpha$ and CypA expressions were associated with shorter OS, while low Caspase 3 expression was associated with shorter OS. In the multivariate analysis, stage, CypA and HIF-1 $\alpha$ continued to be significant poor prognostic factors [41, 42].

This study showed that in BPH cases, a statistically significant correlation was found between HIF-1 $\alpha /$ Cyclophilin A expression. There was an inverse correlation between CypA/ Caspase 3 but not statistically significant. Also, there was an inverse correlation between HIF-1 $\alpha /$ Caspase 3 but not statistically significant. In PIN cases, no statistically significant association detected between examined markers, HIF-1 $\alpha$ / CypA expression, between CypA/ Caspase 3 and HIF-1 $\alpha /$ Caspase 3. In PCa cases, a statistically significant association was noticed between HIF-1 $\alpha /$ CypA expression. An inverse statistically significant association was observed between CypA/ Caspase 3 and HIF-1 $\alpha /$ Caspase 3.

Our study showed a significantly correlation between HIF-1 $\alpha$ expression, CypA expression and negative correlation with Caspase 3 expression in $\mathrm{PCa}$. HIF-1 $\alpha$ was reported to work as a transcriptional coactivator for upregulation of CypA expression in hypoxic situation which in turn inhibits the apoptotic cascade through inhibition of caspase-3. 
Conclusion, our study confirmed that the reduction of apoptosis in $\mathrm{PCa}$ occured through induction of CypA which in turn was released via direct activation of HIF-1 $\alpha$. CypA may play a noble chemotherapeutic goal for managements that could overcome the chemoresistance in tumor cells. We suggest further studies on CypA to realize its mode of actions, which will optimistically result in innovative management against solid tissue tumors.

\section{REFERENCES}

1. Ibrahim A. S., Hussein K. M., N. H. Nabiel, Barka, H., \& Kamel H. (2014). Cancer Incidence in Egypt: Results of the National Population-Based Cancer Registry Program. Journal of Cancer Epidemiology, Article ID 437971, 18 pages.

2. National Institute for Clinical Excellence (UK) clinical guideline 58. (2008). Prostate cancer: diagnosis and treatment; http://www.nice.org. uk/cg58.

3. Rankin, E.B., \& Giaccia, A.J. (2008). The role of hypoxia inducible factors in tumorigenesis. Cell Death Differ, 15: 678-685.

4. Semenza, G.L. (2012). Hypoxia-inducible factors: mediators of cancer progression and targets for cancer therapy. Trends Pharmacol Sci, 33: 207214.

5. Lu, X., \& Kang, Y. (2010). Hypoxia and hypoxiainducible factors: master regulators of metastasis. Clin Cancer Res, 16: 5928-5935.

6. Xiao, L.J., Chen, Y.Y., Lin, P. (2012). Hypoxia increases CX3CR1 expression via HIF-1 and NF$\kappa \mathrm{B}$ in androgen-independent prostate cancer cells. Int J Oncol, 41(5):1827-1836.

7. Handschumacher, R., Harding, M., Rice, J., Drugge, R., Speicher, D. (1984). Cyclophilin: A specific cytosolic binding protein for cyclosporin A. Science, 226: 544-7.

8. Lang, K., Schmid, F.X., Fischer, G. (1987). Catalysis of protein folding by prolyl isomerase. Nature, 329: 268-70.

9. Lee, J. (2010). Role of cyclophilin a during oncogenesis. Arch Pharm Res, 33: 181-7.

10. Lee, J, Kim, S.S. (2010). Current implications of cyclophilins in human cancers. J Exp Clin Cancer Res, 29: 97.

11. Nigro, P., Pompilio, G., Capogrossi, M.C. (2013). Cyclophilin A: A key player for human disease. Cell Death Dis, 4: e888.

12. Zheng, J., Koblinski, J.E., Dutson, L.V., Feeney, Y.B., Clevenger, C.V. (2008). Prolyl isomerase cyclophilin a regulation of Janus-activated kinase2 and the progression of human breast cancer. Cancer Res, 68:7769- 7778

13. Hathout, Y., Riordan, K., Gehrmann, M., Fenselau, C. (2002). Differential protein expression in the cytosol fraction of an MCF-7 breast cancer cell line selected for resistance toward melphalan. $J$ Proteome Res, 1: 435-442

14. Campa, M.J., Wang, M.Z., Howard, B., Fitzgerald,
M.C., Patz, E.F. (2003). Protein expression profiling identifies macrophage migration inhibitory factor and cyclophilin $\mathrm{A}$ as potential molecular targets in non-small cell lung cancer. Cancer Res, 63:1652-1656.

15. Howard, B.A., Furumai, R., Campa, M.J. (2005). Stable RNA interference-mediated suppression of cyclophilin A diminishes non-small cell lung tumor growth in vivo. Cancer Res, 65: 8853-60.

16. Feng W, Xin Y, Xiao Y, Li W, Sun, D. (2015). Cyclophilin A enhances cell proliferation and xenografted tumor growth of early gastric cancer. Dig Dis Sci, 60: 2700-711.

17. Cecconi, D., Astner, H., Donadelli, M., Palmieri, M., Missiaglia, E., Hamdan, M., Scarpa, A., Righetti, P.G. (2003). Proteomic analysis of pancreatic ductal carcinoma cells treated with 5aza-2'deoxycytidine. Electrophoresis, 24:42914303.

18. Obchoei, S., Weakley, S.M., Wongkham, S. (2011). Cyclophilin A enhances cell proliferation and tumor growth of liver fluke associated cholangiocarcinoma. Mol Cancer, 10: 102.

19. Lim, S.O., Park, S.J., Kim, W., Park, S.G., Kim, H.J., Kim, Y.I., Sohn, T.S., Noh, J.H., Jung, G. (2002). Proteome analysis of hepatocellular carcinoma. Biochem Biophys Res Commun, 291:1031-1037

20. Yang, J., Li, A., Yang, Y., Li, X. (2011). Identification of cyclophilin $\mathrm{A}$ as a potential prognostic factor for clear-cell renal cell carcinoma by comparative proteomic analysis, Cancer Biol Ther, 11(5):535-546

21. Melle, C., Osterloh, D., Ernst, G., Schimmel, B., Bleul, A., von, Eggeling, F. (2005). Identification of proteins from colorectal cancer tissue by twodimensional gel electrophoresis and SELDI mass spectrometry. Int J Mol Med, 16: 11 -7.

22. Sun, S., Wang, Q., Giang, A. (2011). Knockdown of CypA inhibits interleukin-8 (IL-8) and IL-8mediated proliferation and tumor growth of glioblastoma cells through down-regulated NF-kB. J Neuro oncol, 101:1 -14.

23. Doyle, V., Virji, S., Crompton, M. (1999). Evidence that cyclophilin-A protects cells against oxidative stress. Biochem J, 341:127-32.

24. O'Neill, A.J., Boran, S.A., O'Keane, C., Coffey, R.N., Hegarty, N.J., Hegarty, P., Gaffney E.F., Fitzpatrick, J.M., Watson, R.W. (2001). Caspase 3 expression in benign prostatic hyperplasia and prostate carcinoma. Prostate, 47:183-188.

25. Ranasinghe, W. K., Xiao, L., Kovac, S., Chang, M., Michiels, C., Bolton, D., ... \& Patel, O. (2013). The role of hypoxia-inducible factor $1 \alpha$ in determining the properties of castrate-resistant prostate cancers. PloS one, 8(1), e54251.

26. Handschumacher, R. E., Harding, M. W., Rice, J., Drugge, R. J., \& Speicher, D. W. (1984). Cyclophilin: a specific cytosolic binding protein for cyclosporin A. Science, 226(4674), 544-547. 
27. Harding, M. W., Handschumacher, R. E., \& Speicher, D. W. (1986). Isolation and amino acid sequence of cyclophilin. Journal of Biological Chemistry, 261(18), 8547-8555.

28. Lu, X., \& Kang, Y. (2010). Hypoxia and hypoxiainducible factors: master regulators of metastasis. Clinical cancer research, 16(24), 59285935.

29. Eckschlager, T., Adam, V., Hrabeta, J., Figova, K., \& Kizek, R. (2009). Metallothioneins and cancer. Current Protein and Peptide Science, 10(4), 360-375.

30. Alves, C. C., Carneiro, F., Hoefler, H., \& Becker, K. F. (2009). Role of the epithelial-mesenchymal transition regulator Slug in primary human cancers. Front Biosci, 14(1), 3035-3050.

31. Storci, G., Sansone, P., Trere, D., Tavolari, S., Taffurelli, M., Ceccarelli, C., ... \& Santini, D. (2008). The basal- like breast carcinoma phenotype is regulated by SLUG gene expression. The Journal of Pathology: A Journal of the Pathological Society of Great Britain and Ireland, 214(1), 25-37.

32. Ping, H., Chen, X.C., Wang, H., Chen, C.H., Zeng, F.C., Lu, G.C. (2004). Expression and significance of HIF-l $\alpha$ in prostate cancer. Chin J Urol, 25(2):88-90.

33. Ananthanarayanan, V., Deaton, R. J., Yang, X. J., Pins, M. R., \& Gann, P. H. (2006). Alteration of proliferation and apoptotic markers in normal and premalignant tissue associated with prostate cancer. BMC cancer, 6(1), 1-9.

34. Wang, N., Dong, C. R., Jiang, R., Tang, C., Yang, L., Jiang, Q. F., ... \& Liu, Z. M. (2014). Overexpression of HIF-1 $\alpha$, metallothionein and SLUG is associated with high TNM stage and lymph node metastasis in papillary thyroid carcinoma. International journal of clinical and experimental pathology, 7(1), 322.

35. Li, M., Zhai, Q., Bharadwaj, U., Wang, H., Li, F., Fisher, W. E., \& Yao, Q. (2006). Cyclophilin A is overexpressed in human pancreatic cancer cells and stimulates cell proliferation through CD147. Cancer, 106(10), 2284-2294.

36. Li, Y., Guo, H., Dong, D., Wu, H., \& Li, E. (2013). Expression and prognostic relevance of cyclophilin A and matrix metalloproteinase 9 in esophageal squamous cell carcinoma. Diagnostic pathology, 8(1), 207.

37. Qian, Z., Zhao, X., Jiang, M., Jia, W., Zhang, C., Wang, Y., ... \& Yue, W. (2012). Downregulation of cyclophilin A by siRNA diminishes non-small cell lung cancer cell growth and metastasis via the regulation of matrix metallopeptidase 9. BMC cancer, 12(1), 1-11.

38. Choi, K. J., Piao, Y. J., Lim, M. J., Kim, J. H., Ha, J., Choe, W., \& Kim, S. S. (2007). Overexpressed cyclophilin A in cancer cells renders resistance to hypoxia-and cisplatin-induced cell death. Cancer research, 67(8), 3654-3662.

39. Sohn, J. H., Kim, D. H., Choi, N. G., Park, Y. E., \& Ro, J. Y. (2000). Caspase- 3/CPP32 immunoreactivity and its correlation with frequency of apoptotic bodies in human prostatic carcinomas and benign nodular hyperplasias. Histopathology, 37(6), 555-560.

40. Winter, R. N., Kramer, A., Borkowski, A., \& Kyprianou, N. (2001). Loss of caspase-1 and caspase-3 protein expression in human prostate cancer. Cancer research, 61(3), 1227-1232.

41. Zhang, H., Chen, J., Liu, F., Gao, C., Wang, X., Zhao, T., ... \& Hao, J. (2014). CypA, a gene downstream of HIF-1 $\alpha$, promotes the development of PDAC. PLoS One, 9(3), e92824.

42. Howard, B. A., Zheng, Z., Campa, M. J., Wang, M. Z., Sharma, A., Haura, E., \& Patz Jr, E. F. (2004). Translating biomarkers into clinical practice: prognostic implications of cyclophilin A and macrophage migratory inhibitory factor identified from protein expression profiles in non-small cell lung cancer. Lung Cancer, 46(3), 313-323. 